\title{
La innovación en los procesos de provisión de personal: una perspectiva para la gestión del talento humano en las organizaciones
}

\author{
Alfredo Augusto Paz Subillaga \\ Rafael Núñez ${ }^{2}$
}

\section{RESUMEN}

Dentro del proceso de provisión de personal en el área de recursos humanos ha existido siempre un rechazo generalizado a poder manejar indicadores macroeconómicos con enfoque laboral, razón por la cual se desarrolló el presente estudio, puesto que existe la necesidad de indicar esa importancia y además demostrar el conocimiento que tienen los gestores del área de recursos humanos en este tema, aplicándolo a un proceso de provisión de personal por medio de la oferta laboral.

En este sentido, es imperante que la preocupación por enlazar estas variables, es decir, los recursos humanos y los indicadores macroeconómicos sea latente, pues en el estadio de la gestión de las personas que trabajan en un área tan importante como la mencionada, deben tener sus parámetros de acción, de modo que estos puedan servir como base para la toma de decisiones importantes para tener al mejor talento humano dentro de las organizaciones y así lograr los objetivos trazados.

El objetivo de la investigación es demostrar la importancia que tiene la provisión de personal, la innovación de sus procesos y los indicadores macroeconómicos laborales en la oferta laboral en un proceso de provisión de personal. Es una investigación de carácter descriptivo y muestra en sus principales resultados que la innovación y el conocimiento de las variables tratadas en los gestores de recursos humanos en las empresas metalmecánicas son aspectos que pueden ayudar a mejorar el proceso de provisión.

Esta investigación es de tipo cuantitativa con propósito descriptivo, en la cual se consideró la muestra total de 16 gerentes de recursos humanos de empresas metalmecánicas, consideradas grandes, que cuentan con más de mil empleados en la Ciudad de Guatemala.

\footnotetext{
${ }^{1}$ Estudiante del Doctorado en Ciencias de la Investigación, Universidad Mariano Gálvez de Guatemala: pazsubillaga@gmail.com

${ }^{2}$ Asesor, Facultad de Ciencias Económicas UNAH, Coordinador Académico Departamento de Administración de Empresas: rafael.nunez@unah.edu.hn
} 
Entre las conclusiones más importantes de esta investigación están que la innovación en los procesos de recursos humanos debe ser entendida como un valor agregado; cuando factores macroeconómicos y microeconómicos se cruzan con los objetivos de una organización, es cuando la gestión de los recursos humanos debe estar alineada de manera estratégica y no debe ser ajeno para los administradores de recursos humanos, manejar aspectos macroeconómicos, pues dentro de la estrategia y la gestión propia de la administración de este rubro debe existir un interés y una conciencia del impacto que pueda causar la información y estadísticas de este tipo en la empresa.

Palabras clave: indicadores macroeconómicos, innovación, proceso de provisión de personal, oferta laboral, gestión de recursos humanos, investigación.

\section{ABSTRACT}

Within the process of staffing in the area of human resources, there has always been a widespread rejection to handle macroeconomic indicators focused in work, which is why the study was developed. There was the need to indicate this importance, as well as to demonstrate the knowledge that the human resources managers have in this area, applying it to a staffing process through job offers.

In this regard, it is imperative that concern for linking these variables, that is, human resources and macroeconomic indicators is latent, as in the managerial stage people who work in such an important area as the one mentioned above should have action parameters in order to be used as a foundation to make important decisions to be able to have the best talent within organizations and, therefore, to be able to achieve the established objectives.

The objective of this research is to demonstrate the importance of staffing, the innovation of its business processes and macroeconomic indicators for job offering during the staffing process. This is a descriptive study and its main results demonstrate that innovation and knowledge of the variables addressed by the human resource managers in the metalworking companies are aspects that can help improve the staffing process.

Keywords: macroeconomic indicators, innovation, staffing process, job offer, human resources management, research. 


\section{INTRODUCCIÓN}

Cuando se habla de gestión de recursos humanos se refiere a un sinnúmero de acciones y actividades preparadas y enfocadas para mejorar el apoyo al personal de una institución determinada, en donde cada una de las personas que integran la organización representan valiosos espacios de inversión monetaria y de tiempo, para que estos puedan ofrecer resultados esperados.

La administración del capital humano pretende, como objetivo básico, contribuir al éxito de la empresa o institución por medio de su incidencia en la estrategia corporativa, impulsar el uso óptimo del talento y contribuir a los resultados financieros, los valores organizacionales y la cultura de la empresa (Werther, Davis y Guzmán, 2014).

Precisamente, cuando se habla de lograr resultados a nivel general es cuando se debe tener en el gestor de los recursos humanos el conocimiento adecuado para tener herramientas que le permitan una mejor orientación para poseer el mejor capital humano dentro de sus filas y así ayudar a mostrar competitividad en la gestión de la organización como tal.

El propósito fundamental de esta investigación es demostrar la importancia que tienen para los gestores de recursos humanos los índices macroeconómicos laborales, los cuales le permiten tener cierta información importante para tomar mejores decisiones, en particular, para el proceso de provisión de personal, pues tanto este conocimiento como la innovación en los procesos ayuda a que lo que se busque en el mercado laboral se pueda encontrar con ciertas consideraciones estratégicas en dicho proceso.

Al tener una mejor lectura y sobre todo una costumbre de comprender que los índices macroeconómicos ayudan a tener un mejor norte para los procesos de provisión y, sobre todo, para poder entablar una mejor relación entre la gestión, el desempeño y sus resultados, será vital que pueda haber un marco de entendimiento y utilización de estos para mejores usos en los recursos humanos.

Hablar sobre este tipo de temas entre los recursos humanos y la macroeconomía no es tan común y mucho menos la investigación en el área de recursos humanos. Por eso, precisamente, al entender que esta área necesita líneas de investigación que la puedan sustentar y ayudar a seguir creciendo es por lo que nace esta inquietud y necesidad de profundizar en algunos tópicos mencionados. 
La investigación se desarrolló en el área de las empresas metalmecánicas de la Ciudad de Guatemala, tomando en cuenta que por aspectos demográficos y de comercio, estas empresas se encuentran en su mayoría en la capital, en donde se ha podido encontrar una fuente de trabajo bastante considerable para el país.

\section{METODOLOGÍA}

El tipo de estudio desarrollado para esta investigación fue cuantitativo con un enfoque descriptivo, pues el objetivo de la investigación versa sobre determinar la importancia de la provisión de personal, la innovación de sus procesos y sus indicadores macroeconómicos laborales en la oferta laboral para el proceso de provisión de personal.

El ámbito de estudio fue la ciudad capital de Guatemala y sus 16 empresas metalmecánicas más grandes (1,000 empleados hacia arriba) y que, por supuesto, contaran con procesos de provisión debidamente establecidos.

La muestra está conformada por las 16 empresas metalmecánicas y sus 16 gerentes de recursos humanos. Se diseñó un instrumento con preguntas dicotómicas enfocadas a obtener la información necesaria y por último una pregunta abierta para que se adhirieran los comentarios extras que quisieran los entrevistados, las cuales no tendrían tabulación por ser comentarios muy generales o sugerencias al tema de investigación.

Respecto a los temas macroeconómicos, se obtuvo de la Comisión Económica para América Latina y el Caribe (CEPAL), datos relevantes que a nivel de Centroamérica pueden ser bastante interesantes para tomar en cuenta al momento de desarrollar un proceso de provisión de personal.

Precisamente, cuando en un país se necesitan datos de tipo de información macroeconómica laboral (ver gráfico 1), se puede orientar una indicación sobre el desempleo actual en el istmo, pues algunos porcentajes son más significativos que otros y esto ayuda a determinar las líneas de acción que deben tener los procesos de contratación de personal y más aún cuando en ciertas áreas geográficas de nuestros países pueda haber opciones de mano de obra que satisfaga las necesidades de una organización. 
Gráfico 1. Tasa de desempleo 2013

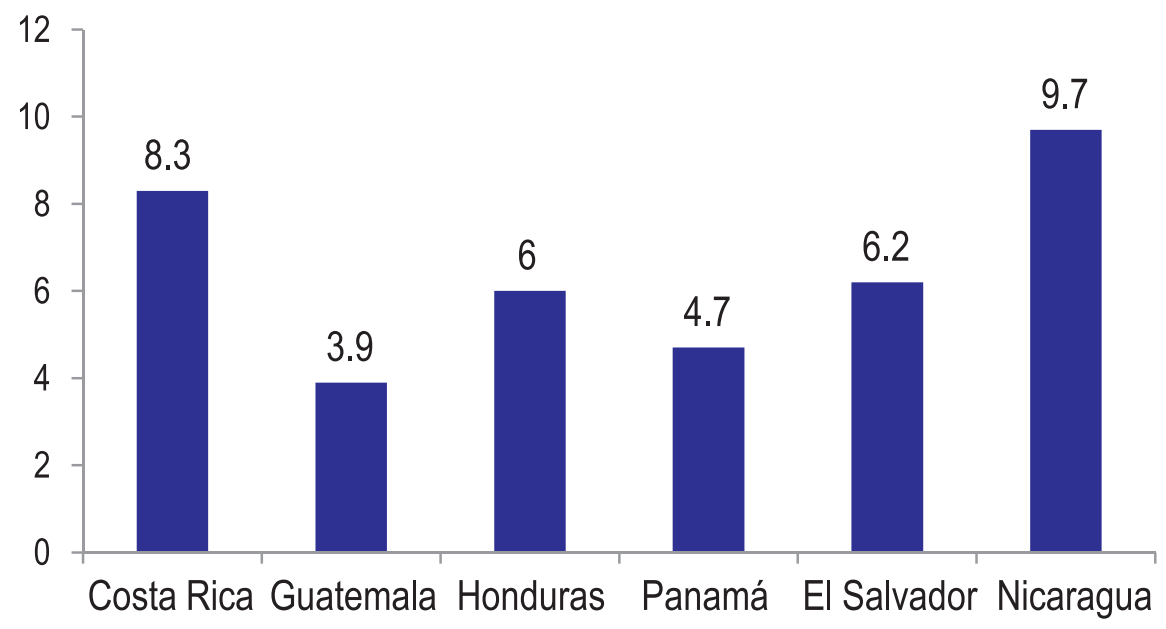

Fuente: CEPALSTAT, 2013.

Gráfico 2. Tasa de alfabetización 15-24 años

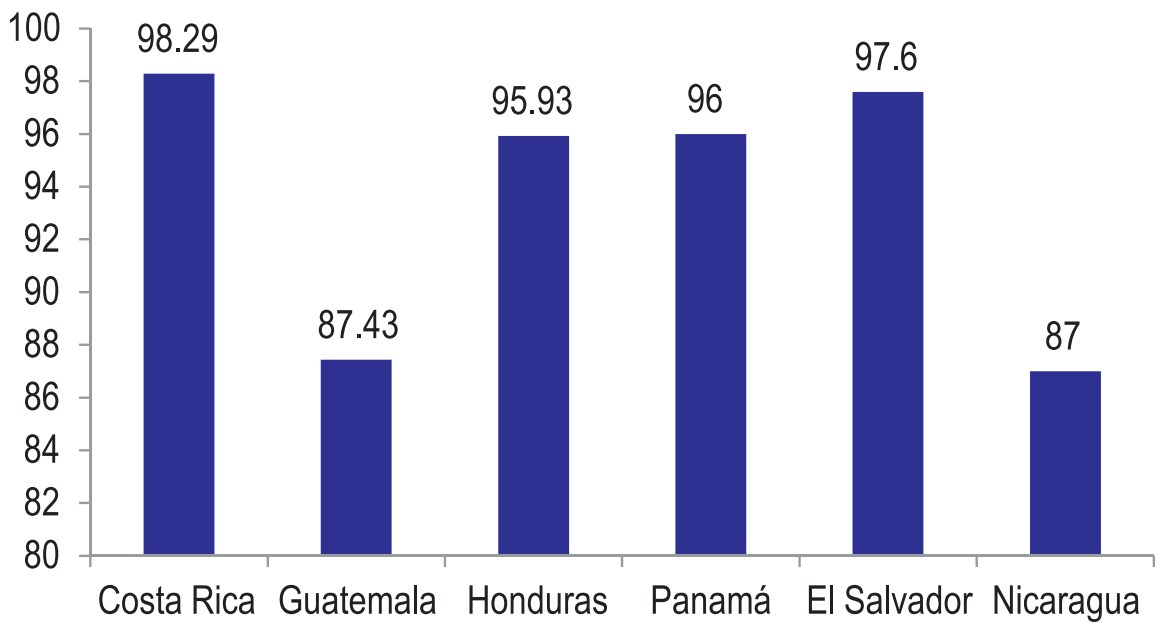

Fuente: CEPALSTAT, 2013.

Definitivamente, en los procesos de provisión de personal y máxime los que son por competencias, deben reflejarse un grupo mínimo de estas para poder contratar al personal adecuado y aspectos como el que se muestra en el gráfico 2 son determinantes al momento de que una institución lucrativa o no pueda o quiera afincar sus intereses en uno o varios países de la región; ya que esto provocará una determinación y, a la vez, cuales son los países punta en este tipo de análisis. 
En la gráfico 3 aparece un dato importante, pues el gasto público en educación se viene a enarbolar en esa oportunidad que pueden tener las personas de escasos recursos de los países para poder formarse y así optar a condiciones laborales, por lo menos, mínimas para su desarrollo social y personal. Sin embargo, los datos no son halagadores precisamente por tener un aspecto social en la parte laboral, el gestor de los recursos humanos y, sobre todo, el responsable del proceso de provisión de personal debe atender ciertas tendencias macroeconómicas que le permitan discernir si en su entorno podrá tener u obtener lo que busca basado en las necesidades organizacionales con que pueda contar el área para la cual esté desarrollándose.

Gráfico 3. Gasto público en educación

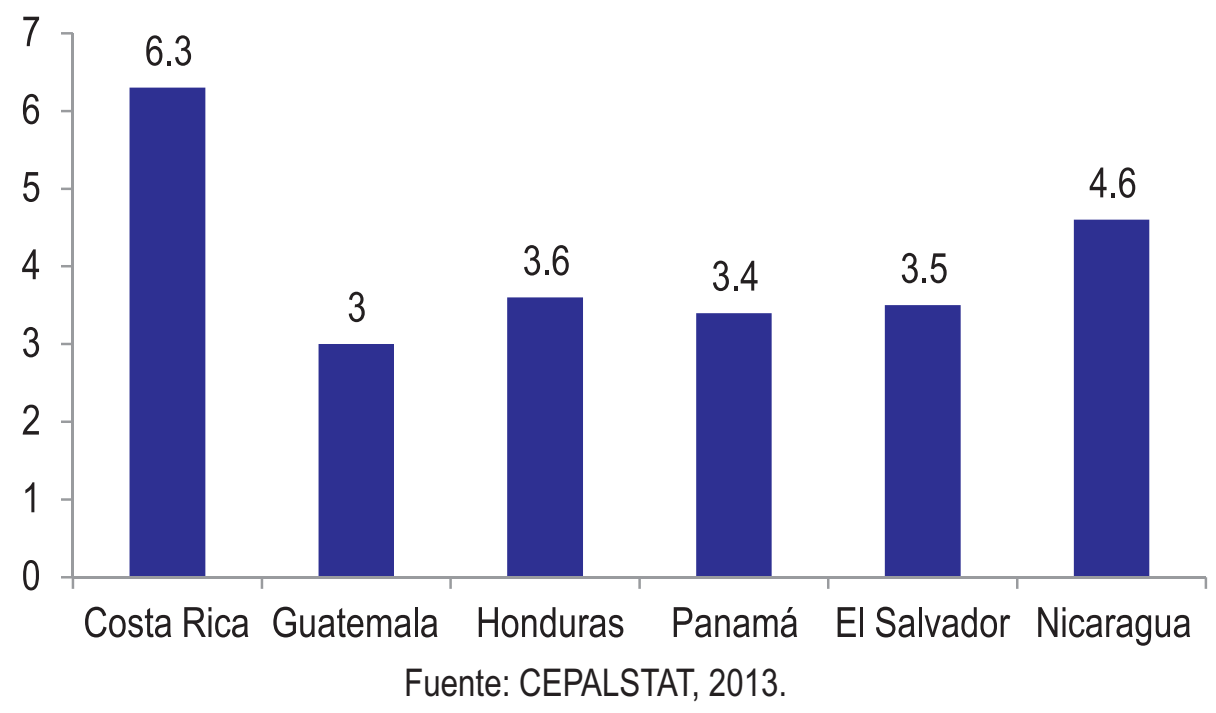

Dentro de los aspectos que se correlacionan en esta investigación está determinar la población urbana ocupada en Centroamérica, como información paralela al aspecto en el que se ha enfocado el trabajo, al área urbana de una ciudad capital centroamericana, por lo que es importante tener esta información y saber cómo está el resto de ciudades de los países del istmo. Esto ayudará a prospectar de mejor manera las acciones a tomar para encauzar las actividades futuras a posibles expansiones de empresas metalmecánicas en la región.

Con el análisis de los aspectos y tablas anteriores, también se hace un llamado a la conciencia del sector laboral y personal de los trabajadores, pues no se pretende que su salario sea utilizado solamente para aspectos como la capacitación, sino que es necesario ir creando esta conciencia tanto en los trabajadores a nivel operativo, como en los estudiantes universitarios que trabajan, puesto que 
mientras más se capaciten, más oportunidades de desarrollo tendrán en sus respectivos países y mientras más se concienticen sobre qué esperar de las empresas que les den el cien por ciento de la capacitación que ellos esperan, habrá que tomar muchas buenas actitudes y motivaciones internas para no caer en aspectos que mermen su desempeño.

Gráfico 4. Población urbana ocupada

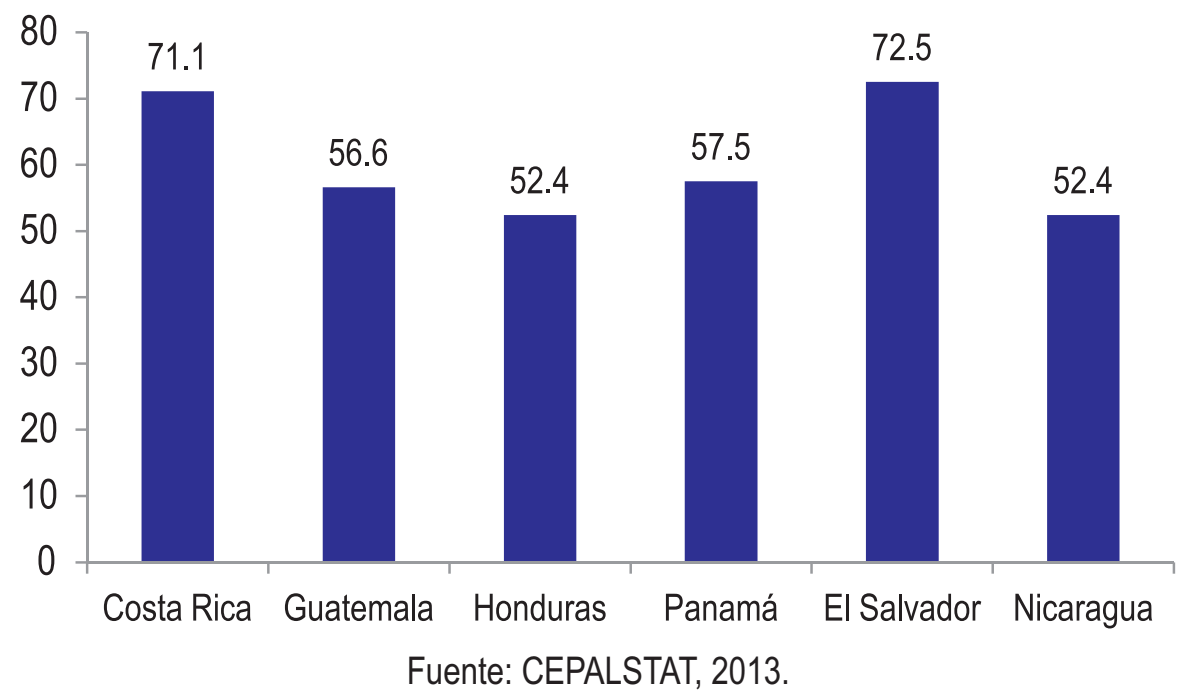

Bajo ningún punto de vista se quiere dar a entender que las empresas o instituciones no capaciten, lo que se quiere es concientizar al trabajador de que aunado a esa capacitación debe haber una preocupación del trabajador para que por sus propios medios pueda darse ese valor agregado que en ocasiones es tan necesario para optar a mejores espacios laborales.

Respecto al área gerencial de los recursos humanos y el presente estudio, se pudo determinar la importancia de manejar estos aspectos para los gerentes de recursos humanos del sector metalmecánico, que deben y pueden ser una guía en su accionar estratégico; por consiguiente, no se puede ocultar la relación de las variables y mucho menos dejar de que estas puedan ser un aporte más que necesario para la toma de decisiones. Como ejemplo se muestran a continuación algunos ejemplos:

1. "El $35 \%$ de las empresas en la región bajo estudio no consiguen el talento necesario para cubrir sus plazas, pero al $23 \%$ de los jóvenes tampoco les interesa obtener un trabajo remunerado" (Prensa Libre, 14 de mayo de 2005, pp.6). 
2. "Pese a la oferta laboral, los jóvenes en Centroamérica y Dominicana carecen de capacitación", según la Cepal, 2014.

3. "...Uno de cada diez jóvenes desempleados no tiene interés en aplicar a una oferta laboral y $73 \%$ de los jóvenes que trabajan se encuentran en búsqueda activa de otro empleo" (Prensa Libre, 14 de mayo de 2005, pp.6).

4. "...Sin embargo, un alto porcentaje... de estos jóvenes indica que existen procesos de reclutamiento de personal que no son atractivos, pues carecen de información para aplicar a plazas que ofrezcan mejores condiciones..." (Prensa Libre, 14 de mayo de 2005,p p.6).

De los ejemplos mostrados anteriormente, se puede determinar que esa necesidad de autocapacitarse y ser competitivo debe volverse en una cultura más que una necesidad, puesto que al tener más conocimientos podrán sentirse cómodos en procesos de provisión de personal que los requieran.

Para las gerencias de recursos humanos y tomando en cuenta el ejemplo anterior, es importante también que parte de la innovación que se busca sea una comunicación clara y concreta sobre lo que puede atenderse como una necesidad latente de las personas que optan a procesos de provisión de personal.

\section{RESULTADOS}

En términos generales, la investigación arroja información importante en donde los gerentes entrevistados señalan ciertos conocimientos sobre aspectos macroeconómicos laborales y su importancia en los procesos de provisión que estos manejan, sin embargo, algunos dicen que tener el conocimiento no les basta, pues no han enlazado esta información con sus respectivos procesos y así poder aprovechar la información necesaria y dar un giro de manera innovadora al momento de ofrecer alguna plaza a algún optante o grupo de optantes a determinados puestos de trabajo.

Es necesario identificar más la importancia de los aspectos macroeconómicos laborales dentro de la gestión de los recursos humanos, puesto que en este estudio y en sus resultados se muestra que el $68.75 \%$ de los gerentes entrevistados sí conocen de estos, pero el fondo es precisamente poder enlazar esos conocimientos con la realidad de los procesos de provisión, por tanto, se puede tener el conocimiento, mas se puede adolecer de la aplicación. 
La disposición de poder aplicar la innovación de procesos de provisión y así mejorar la oferta laboral, se vio reflejada en un $81.25 \%$ de los entrevistados, puesto que se necesita que estos procesos tengan cambios relevantes y a la vez atrayentes para los optantes, ya que no se pueden mantener aspectos añejos y que se pretenda que todavía puedan seguir surtiendo efecto cuando estamos en una época cambiante no solo en la parte tecnológica, sino también en la parte personal y laboral.

Al conjuntar los aspectos macroeconómicos, la provisión de personal y la innovación, basados en la consulta a los gerentes de recursos humanos, se tuvo información importante. En algunos casos las respuestas fueron que sí conocen de los aspectos y además que sí innovan en sus procesos de provisión; así como los casos negativos en donde no se innova, no se conocen aspectos macroeconómicos y mantienen sus procesos de provisión de manera estática. En consecuencia, se necesita que haya una responsabilidad al respecto y se puedan conjugar estas variables, de tal forma que no solamente el optante 0 el gerente sean los beneficiados, sino que el cúmulo de actores principales en este tema sean los beneficiados, es decir, la organización como tal pueda contar con un colaborador comprometido y capacitado, además que pueda ofrecer un gestor que pueda llevar un proceso de provisión acorde a las necesidades de la empresa y, por supuesto, la persona que opta a un puesto tenga la confianza de que puede ingresar a un tipo de empresa que le provea la estabilidad que anda buscando y así se conjuguen objetivos tanto personales como empresariales de manera breve y directa.

Gráfico 5. Conocimientos de aspectos macroeconómicos laborales

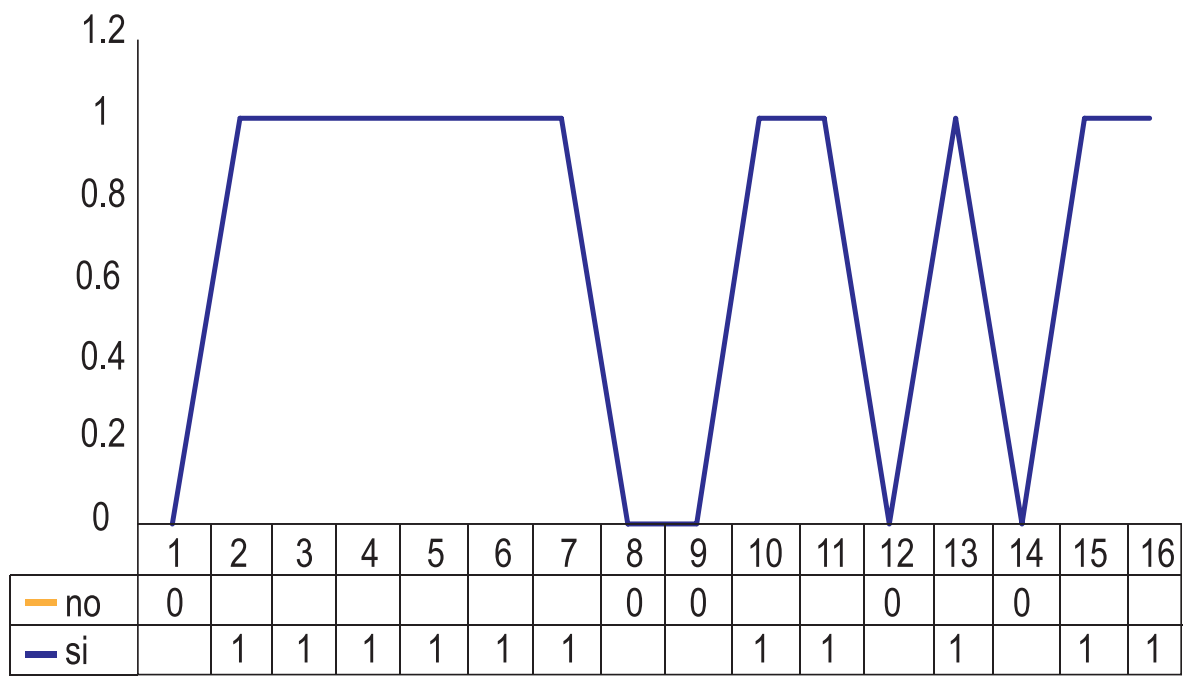

Fuente: elaboración propia. 
Gráfico 6. Disposición de innovar su proceso de provisión para mejorar la oferta laboral

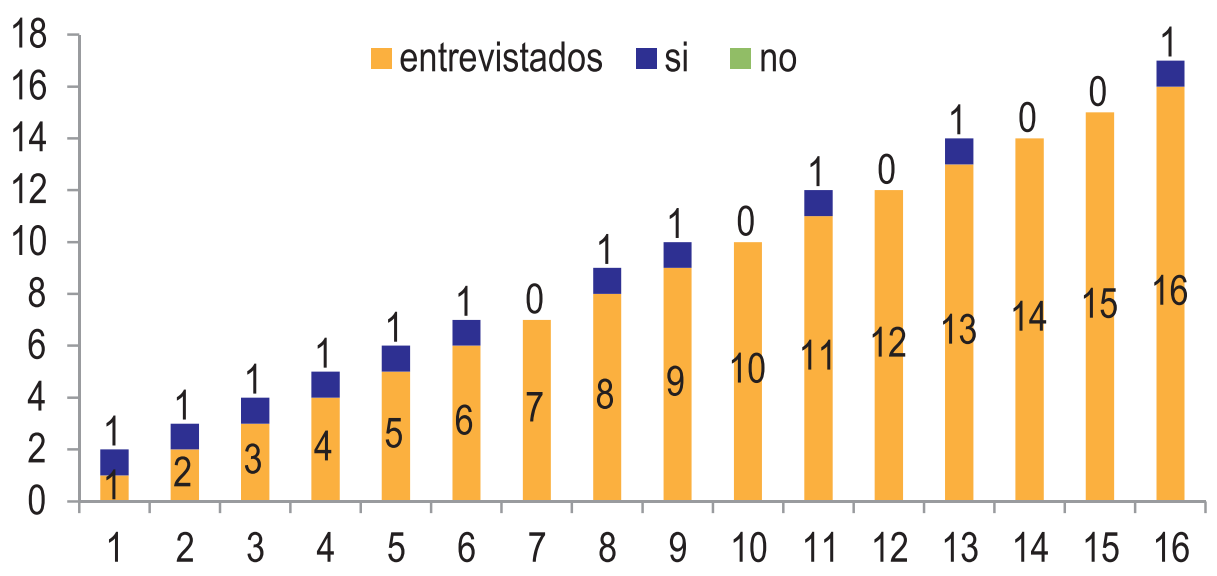

Fuente: elaboración propia.

Gráfico 7. Comparación de aspectos relevantes

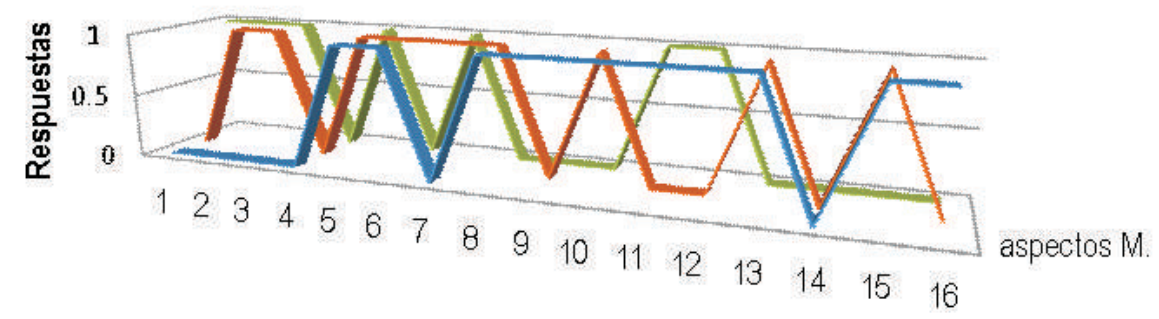

\begin{tabular}{|l|c|c|c|c|c|c|c|c|c|c|c|c|c|c|c|c|}
\cline { 2 - 14 } \multicolumn{1}{c|}{} & 1 & 2 & 3 & 4 & 5 & 6 & 7 & 8 & 9 & 10 & 11 & 12 & 13 & 14 & 15 & 16 \\
\hline - aspectos M & 0 & 0 & 0 & 0 & 1 & 1 & 0 & 1 & 1 & 1 & 1 & 1 & 1 & 0 & 1 & 1 \\
\hline - provisión & 0 & 1 & 1 & 0 & 1 & 1 & 1 & 1 & 0 & 1 & 0 & 0 & 1 & 0 & 1 & 0 \\
\hline - innovación & 1 & 1 & 1 & 0 & 1 & 0 & 1 & 0 & 0 & 0 & 1 & 1 & 0 & 0 & 0 & 0 \\
\hline
\end{tabular}

Fuente: elaboración propia.

\section{CONCLUSIONES}

La innovación en los procesos, no solamente de recursos humanos, sino en general, debe ser entendida y aceptada como un valor agregado, pues representa, en su momento, resultados que buscan las organizaciones para optimizar sus objetivos planteados en un tiempo determinado. 
Precisamente, cuando factores macroeconómicos y microeconómicos se cruzan con los objetivos de una organización, es cuando la gestión de los recursos humanos deben estar alineada con los objetivos generales y específicos de una organización, la cual busca poder afrontar de buena manera estos factores y así tener la capacidad de afrontar sus amenazas y volverlas fortalezas.

La importancia de un proceso de provisión de personal adecuado y bien estructurado es parte del éxito que buscan las organizaciones, lo que permite que la cultura organizacional sea más robusta y pueda sentirse como propia por medio de los colaboradores.

No debe ser ajeno para los administradores de recursos humanos manejar aspectos macroeconómicos, pues dentro de la estrategia y la gestión propia de la administración de este rubro debe existir un interés y una conciencia de que el impacto que pueda causar información y estadísticas de este tipo en la empresa serán relevantes para poder llegar al cumplimiento de objetivos tanto de personal como generales para una organización cualquiera.

\section{AGRADECIMIENTO}

Se agradece el apoyo recibido del Lic. Rafael Núñez Solórzano del Departamento de Administración de Empresas de la Facultad de Ciencias Económicas, del Lic. Oscar Molina del Departamento de Propiedad Intelectual de la Dirección de Investigación Científica y Posgrado; del Lic. Ángel Ávila y del Lic. Héctor Galindo.

\section{BIBLIOGRAFÍA}

Cabarcas Ortega, M. (2008). Gestión del talento humano. Colombia: Universidad de Cartagena.

Cepal. (2013). Anuario estadístico.

Delgado, M. G.; Romero, A. y Vásquez, E. (2009). Gestión de recursos humanos, del análisis teórico a la solución práctica. España: Pearson Prentice Hall.

García Fenton, V. (2011). Procedimiento para la implementación del capital humano. La Habana: Instituto Superior Politécnico José Antonio Echeverría.

Hernández Darias, I. (2011). Gestión del conocimiento en empresas cubanas. Revista avanzada científica, CIGET-MATANZAS, 13.

Hernández Darias, I. y Salazar Fernández, D. (2011). La red capital humano: la experiencia de gestión del conocimiento para sistema de gestión integrada del capital humano en empresas. Redalyc. Org, 15. La Habana. 
Loja, U. (2012). La gestión del talento humano. Ecuador: Facultad de Ciencias Económicas.

Moll, S. (2012). Teorías del desempleo en Guatemala. Política pública. Guatemala: Ministerio de Trabajo de Guatemala.

Rendón, M. (14 de mayo de 2005). Jóvenes centroamericanos y dominicanos carecen de capacitación. Prensa Libre, 6-7.

Werther, W.; Davis, K. y Guzmán, M. (2014). Administración de recursos humanos. Gestión del capital humano. México: Mc Graw Hill. 